\title{
Spontaneous Tumor Lysis Syndrome in a Patient with Metastatic Small Cell Carcinoma of the Lung
}

\author{
Parikshit Padhi* and Shailendra Singh
}

Internal Medicine, West Penn Allegheny Health System, Pittsburgh, Pennsylvania, 15212, USA

\begin{abstract}
Tumor lysis syndrome (TLS) is a life-threatening condition which could result in electrolyte imbalances, acute renal failure, seizures, arrhythmias and sudden death. It is mostly seen after the initiation of chemotherapy in hematological malignancies such as Burkitt's lymphoma and other Non-Hodgkin's Lymphoma. Solid tumors are rarely associated with Tumor Lysis Syndrome. TLS prior to the initiation of chemotherapy is known as spontaneous TLS, of which most of the literature involves hematological malignancies. There have been only a handful of case reports of spontaneous TLS occurring in solid tumors. We are presenting a patient who was newly diagnosed with metastatic small cell cancer of lung who developed spontaneous TLS. To our knowledge this is the first reported case of spontaneous TLS in small cell cancer of the lung. We will also review the literature on spontaneous TLS in solid tumors and whether as clinicians we can identify high-risk patients. Prompt diagnosis is essential in order to initiate necessary prophylactic precautions. After reviewing the few case reports it appears bulky tumors, site of metastases, prior renal functions may predict risk of suffering from TLS in solid tumors.
\end{abstract}

Keywords: Spontaneous tumor lysis syndrome; Small cell cancer of lung; Acute renal failure; Chemotherapy; Solid tumors

\section{Introduction}

Tumor Lysis Syndrome is characterized by an array of metabolic derangements such as hyperuricemia, hyperphosphatemia, hyperkalemia and hypocalcemia. The rapid release of cellular components into the cells after the lysis of malignant cells [1] leads to the above metabolic abnormalities. It is predominantly seen after the initiation of chemotherapy in hematological malignancies and is seldomly seen in solid tumors [1]. We present a case of a patient diagnosed with metastatic small cell carcinoma who developed tumor lysis syndrome prior to the initiation of chemotherapy, which is a rare manifestation of this disease. With a thorough search of Medline, Cochrane and CINAHL databases, to our knowledge this is the first reported case of spontaneous tumor lysis syndrome in small cell carcinoma of the lung.

\section{Case Presentation}

We present a 73 year old Caucasian lady who presented to our hospital with complaints of abdominal distention and bilateral lower extremity swelling that had been ongoing for 2 weeks. At her primary care physicians' clinic she was found to have an ALT of 315, AST of 689, ALP of 294, GGTP of 465 and Lipase of 92 and was sent to our hospital for further evaluation. On further questioning she complained of non-bloody diarrhea that started a week prior to her visit to her family physician. She denied suffering from any recent infections, fever, chills, vomiting, abdominal pain, or ingestion of any new medications. She denies having been diagnosed with any medical illnesses or malignancies in the past. She has never been diagnosed with any renal disease in the past. Apart from a multivitamin she takes no prescribed medications. She had a 50 pack year smoking history and denies abusing IV drugs. Her mother died of a pulmonary embolism and her sister has been diagnosed with lung cancer, the details of which she was not aware of.

On physical examination her vitals were within normal limits. She appeared cachectic. There were no palpable lymph nodes. Her liver was enlarged, hard and nodular. She had a palpable spleen. A moderate amount of ascites and a positive fluid thrill was elicited as well. Her abdomen was non-tender and she had no signs of guarding or rigidity. Her initial labs revealed normal electrolytes and renal function tests (BUN $5 \mathrm{mg} / \mathrm{dL}$ and Creatinine $0.37 \mathrm{mg} / \mathrm{dL}$ ). As mentioned above she had deranged liver enzymes. Her salicylate levels, ethanol levels and hepatitis panel were all within normal limits. Her WBC count on presentation was $9910 / \mathrm{mcL}$, her hematocrit was $35.1 \%$ and Platelet count was $214,000 / \mathrm{mcL}$. The pertinent initial labs are all revealed in Table 1 .

A CT scan of her Thorax and Abdomen revealed a right upper lobe mass of approximately $4 \mathrm{~cm}$ with right hilar and mediastinal lymphadenopathy, and bronchial wall thickening. Her liver was enlarged measuring $19 \mathrm{cms}$ in the cranio-caudal dimension with innumerable subcentimer, hypodense, non-enhancing lesions with no arterial phase enhancement. There was a high suspicion that she was suffering from a metastatic lung malignancy. Once the patient was aware of her findings she requested outpatient follow up. Appointments were made with our Oncology, Pulmonology and Gastroenterology departments.

She failed to make her scheduled appointments and returned to the hospital after 10 days. At this time she was suffering from worsening dyspnea, non-productive cough and abdominal distention. Her liver was tender to palpate and was encompassing her entire abdomen. A repeat CT scan of her Thorax and Abdomen revealed a rapidly progressing right upper lobe mass with post-obstructive atelectasis,

*Corresponding author: Parikshit Padhi M.D, Internal Medicine, West Penn Allegheny Health System, 8 Allegheny Center, Apt 324, Pittsburgh, Pennsylvania, 15212, USA, Tel: 872-800-3481; E-mail: parikshit.padhi@gmail. com, ppadhi@wpahs.org

Received May 10, 2012; Accepted June 18, 2012; Published June 20, 2012

Citation: Padhi P, Singh S (2012) Spontaneous Tumor Lysis Syndrome in a Patient with Metastatic Small Cell Carcinoma of the Lung. J Cancer Sci Ther 4 164-166. doi:10.4172/1948-5956.1000133

Copyright: () 2012 Padhi $\mathrm{P}$, et al. This is an open-access article distributed under the terms of the Creative Commons Attribution License, which permits unrestricted use, distribution, and reproduction in any medium, provided the original author and source are credited. 
moderate right pleural effusion and hepatomegaly with numerable hepatic hypodense lesions. A bronchoscopy was performed to biopsy the lung mass. The pathology and cytopathology analysis of the specimen was positive for small cell carcinoma. A diagnosis of Stage IV small cell carcinoma was made and she underwent the necessary evaluations and preparations required for initiation of chemotherapy. Prior to her first chemotherapy cycle she developed acute kidney injury (creatinine $0.37 \mathrm{mg} / \mathrm{dL}$ to $0.97 \mathrm{mg} / \mathrm{dL}$ ), increase in her potassium levels and hyperphosphatemia (3.6 to $4.7 \mathrm{mmol} / \mathrm{L}$ and 3.2 to $5.1 \mathrm{mg}$ / $\mathrm{dL}$ respectively). Her uric acid was elevated $(14 \mathrm{mg} / \mathrm{dL})$ as well. With all these findings she was diagnosed with tumor lysis syndrome. Aggressive hydration was initiated with close monitoring of her electrolytes. Her respiratory status worsened on that similar day for which she had to be intubated. The family withdrew care the following day and she passed away on Day 4 of her second admission because of acute respiratory failure secondary to bronchial obstruction.

\section{Discussion}

Tumor lysis syndrome (TLS) is an oncological emergency [2] which Cairo and Bishop have classified it into two groups; namely Laboratory Tumor Lysis Syndrome and Clinical Tumor Lysis Syndrome [3] (Table 2). It is most commonly seen after the initiation

\begin{tabular}{|l|c|c|c|}
\hline & $\begin{array}{c}\text { DAY 1 (1 } \\
\text { admission) }\end{array}$ & $\begin{array}{c}\text { DAY 1 (2 } \\
\text { admission) }\end{array}$ & $\begin{array}{c}\text { DAY 4 (2 } \\
\text { admission) }\end{array}$ \\
\hline SODIUM (mmol/L) & 138 & 136 & 140 \\
\hline POTASSIUM (mmol/L) & 3.6 & 4.7 & 4.7 \\
\hline CREATININE (mg/dL) & 0.37 & 0.73 & 0.97 \\
\hline CALCIUM (mmol/L) & 8.7 & 9.2 & 8.8 \\
\hline WBC COUNT (k/mcL) & 9.91 & 10.73 & 13.74 \\
\hline HEMOGLOBIN (g/dL) & 11.6 & 11.5 & 8.9 \\
\hline HEMATOCRIT (\%) & 35.1 & 33.0 & 27.4 \\
\hline PHOSPHORUS (mg/dL) & 3.2 & 5.1 & 5.2 \\
\hline URIC ACID (mg/dL) & & 13.4 & 14.0 \\
\hline LDH (U/L) & 1196 & 1615 & 1470 \\
\hline AST (U/L) & 689 & 857 & 664 \\
\hline ALT(U/L) & 315 & 324 & 257 \\
\hline ALP(U/L) & 294 & 342 & 257 \\
\hline
\end{tabular}

Table 1: The pertinent initial labs.

\section{Laboratory Tumor Lysis Syndrome \\ Hyperuricemia: Uric acid $>8 \mathrm{mg} /$ $\mathrm{dL}(475.8 \mu \mathrm{mol} / \mathrm{L})$ in adults or above the upper limit of the normal range in children}

Hyperphosphatemia: Phosphorus $>4.5 \mathrm{mg} / \mathrm{dL}(1.5 \mathrm{mmol} / \mathrm{L})$ in adults or $>6.5 \mathrm{mg} / \mathrm{dL}(2.1 \mathrm{mmol} / \mathrm{L})$ in children

Hyperkalemia: Potassium $>6 \mathrm{mmol} / \mathrm{L}$ Hypocalcemia: Corrected calcium calcium $<1.12(0.3 \mathrm{mmol} / \mathrm{liter}$ $<7.0 \mathrm{mg} / \mathrm{dl}$ ( $1.75 \mathrm{mmol} / \mathrm{liter})$ or ionized

\section{Clinical Tumor Lysis Syndrome} Cardiac dysrythmia or sudden death probably of definitely caused by hyperkalemia

Cardiac dysrhythmia, sudden death seizure, neuromuscular irritability (tetany, paresthesias, muscle twitching, carpopedal spasm, Trousseau's sign, Chvostek's sign, laryngospasm, or bronchospasm), hypotension, or heart failure probably or definitely caused by hypocalcemia

Increase in the serum creatinine level of $0.3 \mathrm{mg} / \mathrm{dl}$ ( $26.5 \mu \mathrm{mol} /$ liter) (or a single value $>1.5$ times the upper limit of the ageappropriate normal range if no baseline creatinine measurement is available) or the presence of oliguria, defined as an average urine output of $<0.5 \mathrm{ml} / \mathrm{kg} / \mathrm{hr}$ for 6 hours

Table 2: Laboratory Tumor Lysis Syndrome and Clinical Tumor Lysis Syndrome [21]. of chemotherapy in hematological malignancies such as Burkitt's lymphoma, Acute Lymphoblastic Leukemias [4]. TLS can progress to acute kidney failure because of urate nephropathy [5], ARDS [6], seizures or even sudden death due to cardiac arrhythmias [1]. Initial management of TLS involves aggressively hydration the patient $(3 \mathrm{~L} /$ $\mathrm{m}^{2} /$ day), vigilant monitoring of electrolytes every 4-6 hours [7], as well as addition of a hypouricemic agent such as allopurinol. Rasburicase, which makes allantoin (end product of the uric acid metabolization) water soluble, has been proven to be beneficial [7] in the management of TLS. Trials such as The Groupe d'Etude des Lymphomes de l'Adulte Trial on Rasburicase Activity in Adult Lymphoma (GRAAL1) study [8] and Pui et al. [9] have demonstrated that addition of Rasburicase has reduced uric acid level quicker and prevented more people from needing dialysis as compared to Allopurinol. Patient who develops End-stage renal disease or anuria would need to be started on dialysis.

Tumor lysis syndrome in solid tumors is a rare phenomenon. It is more commonly associated with highly chemo-sensitive tumors or in patients with a large tumor burden. The various case reports include malignancies such as small cell cancer of lung, Merkel cell cancer, and aggressive Breast cancers [10-12]. If this event occurs prior to the initiation of chemotherapy it is known as Spontaneous Tumor Lysis Syndrome. After a search of Medline, Cochrane and CINAHL databases ours is the first reported case of spontaneous TLS in small cell carcinoma of the lung.

Spontaneous TLS too is associated more with hematological malignancies such as Burkitt's lymphoma [13], other Non-Hodgkins Lymphoma and aggressive leukemias rather than solid tumors. There have been few case reports where this has occurred in solid tumors namely bulky germ cell tumors, squamous cell cancer of the lung, advanced gastric cancer, breast cancer, metastatic melanoma, and advanced metastatic prostate carcinoma [14-17]. All of these patients presented with elevated LDH, bulky, necrotic tumors, hepatic metastases as well as elevated creatinine on presentation. An elevated WBC count on presentation was documented in a few of these cases as well. Although assessing the risk factors for developing acute tumor lysis syndrome in solid tumors is difficult due to its rarity; there appears to be an association with patients who present with bulky tumors, hepatic metastases, $\mathrm{LDH}>1000 \mathrm{IU} / \mathrm{L}$, rapidly proliferating tumors, extensive bone marrow involvement, renal failure, elevated uric acid, and an elevated white blood cell count $(>50,000$ cells $/ \mu \mathrm{L})$ [13]. Cairo et al. [18] risk stratified patients with leukemias, lymphomas, solid tumors, and bulky tumors. They concluded that solid tumors have a lower potential for developing TLS as compared to hematological malignancies. However bulky or chemosensitive solid tumors, germ-cell tumors and small cell carcinoma of the lung have an intermediate-risk for developing TLS after therapy. This study also added that patients with chronic kidney diseases would increase the risk for developing TLS. However this study did not discuss the risk for developing spontaneous tumor lysis syndrome. Montesinos et al. [19] derived a scoring system with parameters of WBC count, LDH and uric acid levels to assess a prognostic indicator of clinical TLS in AML patients [18,19]. Vaisban et al. [20] reported three cases of spontaneous TLS (malignant pheochromocytoma, hepatocellular carcinoma and colon carcinoma) in a 9 month period. I feel this is important as we may be seeing a rise in the incidence of spontaneous TLS in solid tumors. With the advent of newer and more efficacious chemotherapeutic agents the incidence in solid tumors may potentially increase.

Isolated reports of TLS after TACE therapy [21] or initiation 
of Bisphosphonates [22] too have been reported. It is unclear as to whether TACE or Bisphosphonates puts a patient at higher risk as there have been no further reports since.

Our patient had a uric acid $>8 \mathrm{mg} / \mathrm{dL}$, phosphorus of $>4.5 \mathrm{mg} / \mathrm{dL}$. She did develop acute kidney injury as her creatinine increased by more than $0.3 \mathrm{mg} / \mathrm{dL}$ [23]. As she had two of the metabolic derangements and acute kidney injury she had clinical tumor lysis syndrome [24]. Our patient had a metastatic, highly chemosensitive tumor with a high tumor burden. She also had an elevated LDH and liver metastases. All these findings put her at a higher risk of developing TLS. Prophylactically starting her on Allopurinol and aggressive hydration [7] may have prevented her from developing TLS. It may not have prevented her mortality as she died of respiratory failure secondary to extrinsic bronchiolar obstruction from her tumor rather than from the TLS.

Need the presence of two metabolic derangements for diagnosis of Laboratory TLS and for a diagnosis of Clinical TLS one needs Laboratory TLS plus one of the three clinical criteria.

\section{Conclusion}

In conclusion, TLS is an oncological emergency that should be promptly identified and requires aggressive management as it is reversible if treated early. It's incidence is higher in hematological malignancies but bulky tumors or chemosensitive tumors have a high predilection towards developing TLS after initiation of chemotherapy. Although there are studies in patients with AML or other leukemias and lymphomas, it is difficult to risk stratify patients to develop acute or spontaneous TLS. By reviewing the limited case reports available it appears that patients suffering from solid tumors who present with azotemia, significantly elevated LDH (>1000 IU/L), bulky tumors, hepatic metastases, bony metastases or rapidly growing primary tumor are at greater risk. Since its incidence is low, the benefits of TLS prophylaxis in this population are unknown. Its incidence may be rising and as clinicians we should be vigilant in identifying the higher risk patients firsthand. After identification we should keep a close monitor on their electrolytes, renal function, $\mathrm{LDH}$ and uric acid levels. A low threshold should be kept for initiation of TLS prophylaxis in the high-risk population.

\section{References}

1. Hochberg J, Cairo MS (2008) Tumor lysis syndrome: current perspective Haematologica 93: 9-13.

2. Riccio B, Mato A, Olson EM, Berns JS, Luger S, et al. (2006) Spontaneous tumor lysis syndrome in acute myeloid leukemia: two cases and a review of the literature. Cancer Biol Ther 5: 1614-1617.

3. Cairo MS, Bishop M (2004) Tumour lysis syndrome: new therapeutic strategies and classification. Br J Haematol 127: 3-11.

4. Yazigi F, Kolluru A, Al-Katib AM (2011) Tumor Lysis Syndrome as a Consequence of Chronic Lymphocytic Leukemia Treatment With Fludarabine and Rituximab. J Med Cases 2: 188-189.

5. Stapleton FB, Strother DR, Roy S 3rd, Wyatt RJ, McKay CP, et al. (1988) Acute renal failure at onset of therapy for advanced stage Burkitt lymphoma and $B$ cell acute lymphoblastic lymphoma. Pediatrics 82: 863-869.

6. Marenco JP, Nervi A, White AC (1998) ARDS associated with tumor lysis syndrome in a patient with non-Hodgkin's lymphoma. Chest 113: 550-552.

7. Tosi P, Barosi G, Lazzaro C, Liso V, Marchetti M, et al. (2008) Consensus conference on the management of tumor lysis syndrome. Haemotologica 93: 1877-1885.

8. Coiffier B, Mounier N, Bologna S, Fermé C, Tilly H, et al. (2003) Efficacy and safety of rasburicase (recombinant urate oxidase) for the prevention and treatment of hyperuricemia during induction chemotherapy of aggressive nonHodgkin's lymphoma: results of the GRAAL1 (Groupe d'Etude des Lymphomes de l'Adulte Trial on Rasburicase Activity in Adult Lymphoma) study. J Clin Oncol 21: 4402-4406.

9. Pui CH (2002) Rasburicase: a potent uricolytic agent. Expert Opin Pharmacother 3: 433-442.

10. Kalemkerian GP, Darwish B, Varterasian ML (1997) Tumor lysis syndrome in small cell carcinoma and other solid tumors. Am J Med 103: 363-367.

11. Dirix LY, Prove A, Becquart D, Wouters E, Vermeulen $P$, et al. (1991) Tumor lysis syndrome in a patient with metastatic Merkel cell carcinoma. Cancer 67: 2207-2210.

12. Stark ME, Dyer MC, Coonley CJ (1987) Fatal acute tumor lysis syndrome with metastatic breast carcinoma. Cancer 60: 762-764.

13. Shenoy C (2009) Acute spontaneous tumor lysis syndrome in a patient with squamous cell carcinoma of the lung. QJM 102: 71-73.

14. Woo IS, Kim JS, Park MJ, Lee MS, Cheon RW, et al. (2001) Spontaneous acute tumor lysis syndrome with advanced gastric cancer. J Korean Med Sci 16: $115-118$.

15. Sklarin NT, Markham M (1995) Spontaneous recurrent tumor lysis syndrome in breast cancer. Am J Clin Oncol 18: 71-73.

16. Castro MP, VanAuken J, Spencer-Cisek P, Legha S, Sponzo RW, et al. (1999) Acute tumor lysis syndrome associated with concurrent biochemotherapy of metastatic melanoma: a case report and review of the literature. Cancer 85: 1055-1059.

17. Lin CJ, Hsieh RK, Lim KH, Chen HH, Cheng YC, et al. (2007) Fatal spontaneous tumor lysis syndrome in a patient with metastatic, androgen-independent prostate cancer. South Med J 100: 916-917.

18. Cairo MS, Coiffier B, Reiter A, Younes A (2010) Recommendations for the evaluation of risk and prophylaxis of tumour lysis syndrome (TLS) in adults and children with malignant diseases: an expert TLS panel consensus. $\mathrm{Br} \mathrm{J}$ Haematol 149: 578-586.

19. Montesinos P, Lorenzo I, Martín G, Sanz J, Pérez-Sirvent ML, et al. (2008) Tumor lysis syndrome in patients with acute myeloid leukemia: identification of risk factors and development of a predictive model. Haematologica 93: 67-74.

20. Vaisban E, Braester A, Mosenzon O, Kolin M, Horn Y (2003) Spontaneous tumor lysis syndrome in solid tumors: really a rare condition? Am J Med Sc 325: 38-40.

21. Hsieh PM, Hung KC, Chen YS (2009) Tumor lysis syndrome after transarteria chemoembolization of hepatocellular carcinoma: case reports and literature review. World J Gastroenterol 15: 4726-4728.

22. Kurt M, Onal IK, Elkiran T, Altun B, Altundag K, et al. (2005) Acute tumor lysis syndrome triggered by zoledronic Acid in a patient with metastatic lung adenocarcinoma. Med Oncol 22: 203-206.

23. Lattanzio MR, Kopyt NP (2009) Acute kidney injury: new concepts in definition, diagnosis, pathophysiology, and treatment. J Am Osteopath Assoc 109: 13-19.

24. Howard SC, Jones DP, Pui CH (2011) The tumor lysis syndrome. N Engl J Med 364: 1844-1854 